Ann. Zootech., I967, 16 (2), I5I-I64.

\title{
L'ALLAITEMENT ARTIFICIEL DES AGNEAUX
}

\author{
II. - COMPARAISON ENTRE TROIS MODES D'ÉLEVAGE DES AGNEAUX
}

\author{
R. PINOT* et P. MAULÉON \\ avec la collaboration technique de C. CORNu et G. Didier \\ Station de Recherches sur l'Élevage des Ruminants, \\ Centre de Recherches zootechniques et vétérinaires sur les Ruminants, \\ 63 - Theix, près Clermont-Ferrand \\ Laboratoire de Recherches sur la Physiologie de la Reproduction, \\ Centre de Recherches zootechniques, et vétérinaires 37 - Nouzilly \\ Institut national de la Recherche agronomique
}

\section{SOMMAIRE}

Nous avons comparé, dans deux troupeaux, trois modes d'élevage des agneaux : élevage classique sous la mère (lot $\mathrm{A}$ ), élevage sous la mère et sevrage à 8 semaines (lot $\mathrm{B}$ ), allaitement artificiel et sevrage à 8 semaines (lot $\mathrm{C}$ ).

Les agneaux allaités artificiellement ont reçu un "lait " reconstitué à partir d'un aliment d'allaitement comportant $70 \mathrm{p}$. 100 de poudre de lait écrémé et $30 \mathrm{p}$. 100 de suif, dilué à raison de $200 \mathrm{~g} /$ litre ; les trois lots ont reçu les mêmes aliments solides, dans les mêmes conditions.

Les agneaux élevés artificiellement ont effectué pendant la phase d'allaitement des gains de poids comparables à ceux des agneaux doubles élevés sous la mère (tabl. 2 ) ; le sevrage n'a pas ralenti leur croissance, et ils ont fourni des carcasses de bonne qualité. En revanche, les agneaux élevés sous la mère et sevrés précocement à huit semaines ont effectué pendant la phase d'engraissement des gains de poids sensiblement plus faibles que pendant la période d'allaitement; ils ont fourni des carcasses de moins bonne qualité. faisantes.

Cet essai confirme la possibilité d'élever artificiellement des agneaux dans des conditions satis-

\section{INTRODUCTION}

Un certain nombre d'essais récents ont montré qu'il était possible d'élever des agneaux en les allaitant artificiellement dès les premiers jours suivant la naissance, et de leur assurer une vitesse de croissance élevée (Cunningham et al., Ig6r ; PRIBICEVIC et al., I962; WELCH et al., Ig62; LARGE I965 a PINOT et TEISSIER, I965).

\footnotetext{
* Adresse actuelle : C. O.F. N. A., 25 rue du Rempart, 37 -Tours.
} 
La composition (WELCH et al., I962 ; LARGE, I965 $a$; Pinot et TEIssier, I965), et le taux de dilution (LARGE, Ig65 $b$; PINOT, I966) de l'aliment d'allaitement, ont une influence importante sur la vitesse de croissance des agneaux qui reste cependant inférieure à celle d'agneaux recevant du lait de brebis. Le nombre des distributions du " lait », et leur répartition au cours de la journée, semblent avoir également une influence sur la quantité de " lait " ingérée par l'agneau, donc sur sa vitesse de croissance puisque celle-ci est en relation étroite pendant les premières semaines, avec la quantité d'aliment d'allaitement ingérée (LARGE, I965a; PINOT et TEISSIER, I965).

L'allaitement artificiel étant coûteux, il est nécessaire de réduire sa durée en sevrant précocement les agneaux ; ceux-ci peuvent effectuer après le sevrage presque les mêmes gains de poids que les agneaux doubles élevés sous la mère (BROWN, I964) dans la mesure où le sevrage a été assez tardif pour ne pas entraîner de choc trop brutal (CHARLE'T-LÉRY, et al., I954), et oì l'infection parasitaire a pu être limitée (BROWN, I964).

Au cours d'essais antérieurs (PINOT et TEIssier, I965; PrNot, I966), nous avons étudié une technique d'allaitement artificiel permettant à des agneaux croisés Frisons $\times$ Préalpes, sevrés à 5 ou 7 semaines, d'effectuer des gains de poids assez élevés (200-220 g/j) jusqu'à l'âge de 9 semaines.

Dans le présent essai, de caractère plus global, nous avons comparé l'élevage artificiel des agneaux à l'élevage classique sous la mère ; en particulier, nous avons étudié dans quelle mesure des agneaux allaités artificiellement peuvent compenser pendant la phase d'engraissement le retard pris par rapport à des agneaux élevés sous la mère, et fournir une carcasse de qualité analogue. Nous avons tenté de séparer l'effet propre de l'allaitement artificiel de celui du sevrage précoce, en sevrant un troisième lot d'agneaux élevés jusque-là sous la mère, à un âge comparable à celui des agneaux élevés artificiellement; cette dernière technique est đéjà utilisée par les éleveurs qui cherchent à accroître la fréquence des agnelages dans leurs troupeaux.

Ces trois lots d'agneaux ont reçu les mêmes aliments solides, dans les mêmes conditions ; la plupart des mâles ont été abattus, à un même poids. Nous avons pu ainsi étudier l'influence du type d'élevage des agneaux sur leurs gains de poids, la qualité de leurs carcasses et les quantités d'aliments ingérées.

\section{MATÉRIEL, E'T MÉTHODE}

Nous avons étudié des agneaux issus de deux troupeaux de race Préalpes $d u$ Sud, élevés l'un au C. N. R. Z., à Jouy-en-Josas (Yvelines), l'autre au C. R. Z. V. de l'Orfrasière, près de Tours (Indre-et-Loire); ces deux troupeaux avaient néanmoins une origine commune, le troupeau de Tours ayant été récemment constitué avec des agnelles nées dans le troupeau de Jouy.

Ces deux troupeaux ont été utilisés pour la mise au point d'un traitement hormonal permettant d'induire un retour en chaleur des brebis après la mise bas, en vue notamment de préciser l'influence de la présence ou de l'absence de l'agneau ; cet essai a fait l'objet d'un premier compte rendu (MAULEON et $a l ., 1965)$.

La comparaison des trois modes d'élevage des agneaux a été faite en annexe de cet essai.

Dès la mise bas, nous avons constitué dans chaque troupeau trois lots de brebis, en tenant compte de la date d'agnelage et de la taille de la portée. Les brebis de ces trois lots ont allaité leurs agneaux soit jusqu'à r 20 jours, soit jusqu'à 7-9 semaines, ou ont été taries $2-4$ jours après la mise bas. 
Les agneaux correspondant à ces trois lots de brebis ont donc été élevés suivant des modes différents :

- lot $A$ : agneaux élevés sous la mère, et sevrés à r zo jours;

- lot $B$ : agneaux élevés sous la mère, et sevrés précocement à 8 semaines;

- lot $C$ : agneaux séparés de leurs mères 2-4 jours après leur naissance, allaités artificiellement et sevrés précocement à 6 semaines.

Le caractère annexe de cet essai explique que les trois lots d'agneaux n'aient pas été strictement comparables : c'est ainsi que, à Jouy, la production laitière des brebis des lots $\mathrm{A}$ et $\mathrm{B}$ a été très différente, puisque, avant le sevrage, les agneaux du lot $\mathbf{B}$ ont eu un gain de poids inférieur à celui des agneaux correspondants du lot A (fig. I).

De plus, il est difficile de comparer globalement les trois lots en raison des conditions d'élevage qui étaient différentes dans les deux troupeaux. En effet, à Tours, les brebis étaient en majorité primipares et ont donc eu une proportion d'agneaux doubles, et une production laitière, plus faibles que les brebis de Jouy. D'autre part, les conditions générales de l'élevage étaient moins bonnes à Tours, comme le montrent les différences entre les vitesses de croissance des deux lots d'agneaux élevés artificiellement (lot $\mathrm{C}$ ).

Enfin pour des raisons matérielles, il n'a pas été possible de sevrer les agneaux du lot B au même âge que les agneaux allaités artificiellement : s'ils avaient été sevrés à 6 semaines, au lieu de 8, les agneaux du lot B auraient sans doute accusé un ralentissement beaucoup plus marqué de leur croissance.

\section{Animaux}

L'agnelage s'est déroulé du 5 au 25 mars $1_{9} 65$ à Jouy, du 3 mars au 2 avril à Tours. Les agneaux ont été pesés dans les 12 heures suivant la mise bas, et répartis au fur et à mesure des naissances en trois lots. Nous avons ainsi constitué dans chaque troupeau trois lots de 30 à 35 agneaux à Jouy, de 25 à 30 agneaux à Tours (tabl. I).

Les agneaux du lot $\mathrm{A}$ ont été maintenus avec leurs mères, à Jouy dans un hangar ouvert sur un côté, à Tours dans une bergerie close ; ils ont reçu leurs aliments dans une partie de la case où les brebis n'avaient pas accès. Ces agneaux ont été sevrés à l'âge de I 20 jours en moyenne.

Les agneaux du lot $B$ ont été élevés comme ceux du lot A jusqu'au sevrage, à l'âge de 60 jours à Jouy, de 52 jours à Tours; ils ont été dès lors maintenus dans des cases séparées, dans le même bâtiment que leurs mères.

Les agneaux élevés artificiellement (lot $\mathrm{C}$ ) ont été maintenus dans des locaux clos, où la température a pu être maintenue relativement constante, par chauffage central à Jouy, avec des lampes à rayons infrarouges à Tours. Au fur et à mesure des naissances, nous avons constitué trois groupes de 8-ro agneaux, afin de limiter la compétition entre animaux d'âges trop différents, et de permettre un meilleur contrôle des quantités d'aliments ingérées.

Les agneaux mâles ont été retirés de l'expérience lorsqu'ils ont atteint $35 \mathrm{~kg}$, et en majorité abattus ; les agnelles ont été retirées au poids de $30 \mathrm{~kg}$.

Les agneaux ont été pesés toutes les semaines à la même heure, et pendant trois jours consécutifs avant leur sortie de l'expérience; la moyenne de cette triple pesée a servi à déterminer le poids vif à la fin de l'expérience.

\section{Aliments}

Les agneaux du lot $\mathrm{C}$ ont reçu un lait de remplacement préparé en diluant à raison de $200 \mathrm{~g} /$ litre un aliment d'allaitement comportant $3^{\circ} \mathrm{p}$. Ioo de suif et $70 \mathrm{p}$. Io० de poudre de lait écrémé : ce " lait » avait déjà été utilisé lors d'une expérience antérieure (PINOT, r966) et a été préparé de manière identique.

Le " lait " a été distribué à volonté, en quatre repas (6 heures, to heures, $13 \mathrm{~h} 30$, i $7 \mathrm{~h} 30$ ) jusqu'à la $4^{\mathrm{e}}$ semaine; le nombre de repas a été ensuite limité à trois ( 6 heures, Io heures, I 8 heures) jusqu'au milieu de la $6^{\mathbf{e}}$ semaine où il a été alors réduit à deux, puis à un, de manière à sevrer les agneaux à l'âge de six semaines. Les quantités de "lait "ingérées ont été mesurées pour chaque agneau, à chaque repas.

Les agneaux des trois lots ont consommé à volonté un aliment concentré et du foin, de la $2^{\mathrm{e}}$ semaine à la fin de l'expérience. Ils ont reçu les mêmes aliments concentrés à Jouy et à Tours : de 2 à 8 semaines, ils ont reçu un aliment concentré comportant $53 \mathrm{p}$. ıoo d'orge, Io p. Ioo d'avoine, Io p. Ioo de blé, I 2 p. Ioo de tourteau de lin (extraction), Io p. Ioo de tourteau de soja cuit, 5 p. roo de complément minéral (65 p. 100 de phosphate bicalcique, $35 \mathrm{p}$. 100 de sel marin), et 0,5 g de soufre pour soo kg d'aliment. A partir de 9 semaines, ils ont reçu un aliment concentré comportant $50 \mathrm{p}$. roo d'orge, $35 \mathrm{p}$. roo de maïs, ıo $\mathrm{p}$. Ioo de son, $5 \mathrm{p}$. Ioo de complément minéral $65 \cdot 35$. Le passage d'un aliment concentré à l'autre a été réalisé progressivement au cours de la $9^{\mathrm{e}}$ semaine.

Annales de Zootechnie. - I967. 


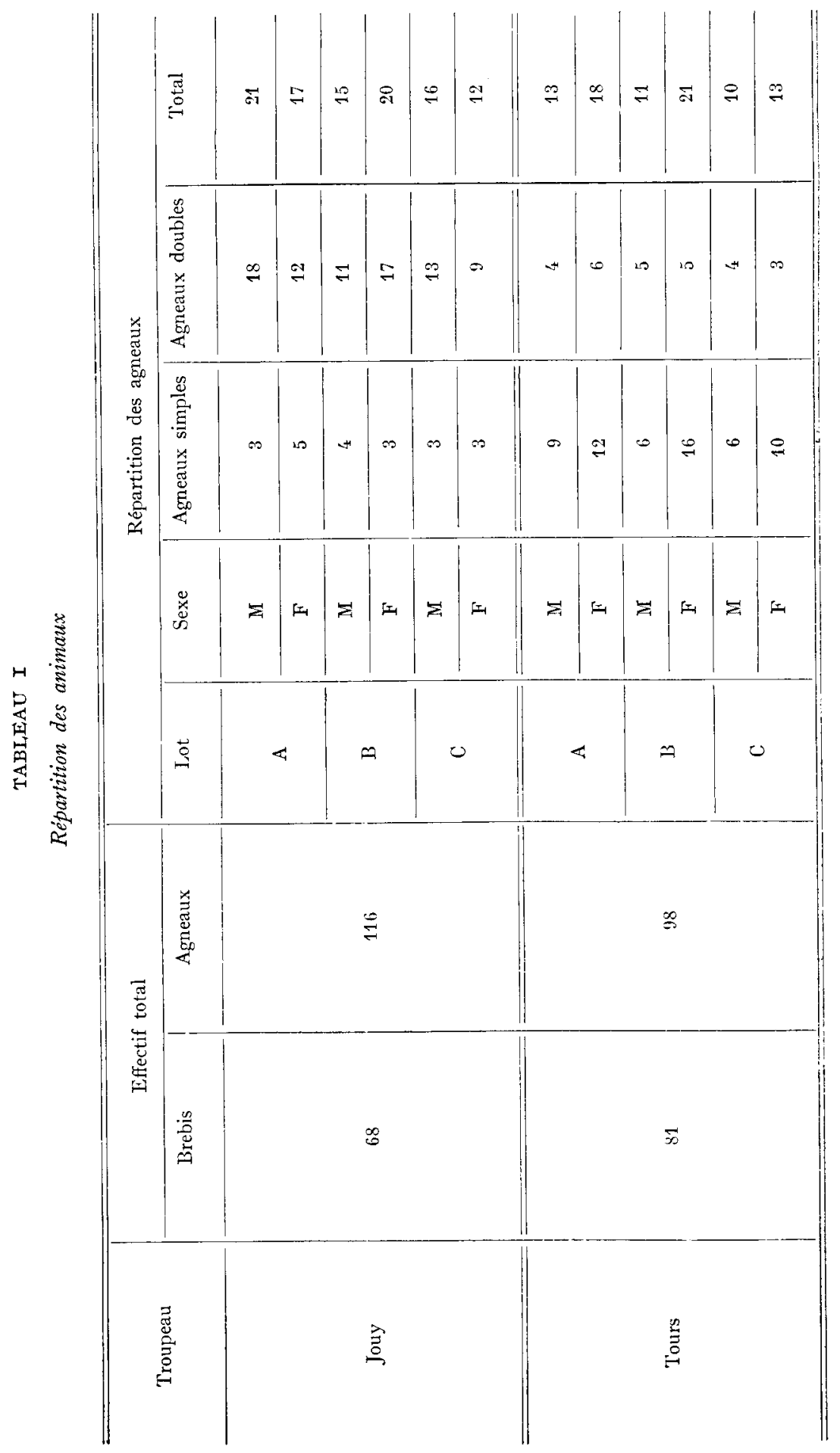


A Jouy, les agneaux ont reçu à partir de la $2^{\mathrm{e}}$ semaine, du foin de luzerne de bonne qualité (i 6,0 p. 10o de matières azotées totales, 37,2 p. Ioo de cellulose brute); à 'Tours, ils ont reçu de la ${ }_{2}^{\mathrm{e}}$ a la $10^{\mathrm{e}}$ semaine un foin comportant de la luzerne et du trèfle violet, de qualité extrêmement variable, et à partir de la $10^{\mathrm{e}}$ semaine, du foin de prairie naturelle, de bonne qualité.

J.es quantités d'aliments distribuées et refusées ont été mesurées chaque jour, jusqu'à la $\mathrm{I}_{4} \mathrm{e}$ semaine à Jouy, jusqu'à la $\mathrm{I}^{\mathrm{e}}$ semaine à Tours. La teneur en matière sèche des aliments offerts et refusés a été mesurée chaque semaine.

\section{Abaltage}

A la fin de l'expérience, un certain nombre d'agneaux, à Jouy ( 15 sur 52) comme à Tours (9 sur 34) ont été conservés, soit conme reproducteurs, soit pour des expériences ultérieures ; ils ont été choisis parmi les agneaux ayant effectué respectivement les gains de poids les plus élevés et les plus faibles. Le nombre d'agneaux abattus dans chaque lot figure dans le tableau 3.

Après l'abattage nous avons pesé le contenu du tube digestif, de manière à connaître le poids vif vide des agneaux. Les carcasses ont été pesées, puis ont fait l'objet des mensurations suivantes : longueur $(\mathrm{K})$, largeur aux trochanters $(\mathrm{G})$, largeur de la poitrine $(\mathrm{Wr})$, profondeur de poitrine $(\mathrm{Th})$, longueur de gigot $(\mathrm{F})$. La graisse périrénale a été détachée et pesée, de manière à avoir une estimation de l'état d'engraissement (BOCCARD et DuMOnT, I960). Chaque carcasse a fait l'objet d'une cotation subjective, portant sur la conformation générale et l'état d'engraissement.

Nous avons choisi pour chaque lot d'agneaux élevés à Jouy, trois carcasses dont le poids et l'état d'engraissement se rapprochaient de la moyenne du lot : elles ont été fendues et les demi-carcasses droites ont été découpées suivant la découpe de référence définie par BoccaRD et DuMoNT (1955) ; chaque morceau a été disséqué, et nous avons séparé et pesé les os, les muscles et les tissus adipeux.

\section{RÉSULTATS}

Les difficultés sanitaires ont été très réduites dans l'un et l'autre troupeau. Nous avons cependant observé pendant la phase d'allaitement quelques cas de diarrhée chez les agneaux allaités artificiellement ( $\operatorname{lot} C)$; deux agneaux de ce lot sont morts à Jouy, l'un accidentellement, l'autre d'entétotoxémie de la caillette (" météorisation ") ; les résultats concernant ces agneaux ont été éliminés.

\section{Gain de poids}

Les courbes moyennes de croissance des agneaux des différents lots sont rapportées dans la figure $I$; les gains de poids moyens journaliers de la naissance à l'âge de 8 semaines d'une part, de l'âge de 8 semaines à la fin de l'expérience d'autre part, sont indiqués dans le tableau 2.

Nous avons distingué dans les lots $\mathrm{A}$ et $\mathrm{B}$, les mâles des femelles, les simples des doubles; dans les lots $\mathrm{C}$, nous n'avons pas distingué les simples des doubles puisque tous les agneaux recevaient le "lait " à volonté. I a proportion de naissances gémellaires ayant été très différente à Jouy et à Tours, nous comparerons essentiellement les résultats concernant les agneaux doubles à Jouy, les agneaux simples à Tours.

Dans les deux troupeaux, les agneaux élevés sous la mère (lot $A$ ) ont eu tout au long de l'expérience une croissance régulière, plus rapide pour les mâles que pour les femelles, pour les simples que pour les doubles. De plus, les agneaux de Jouy ont effectué des gains de poids supérieurs à ceux de Tours.

I,es agneaux du lot $\mathrm{B}$ ont eu à Jouy une vitesse de croissance de la naissance 


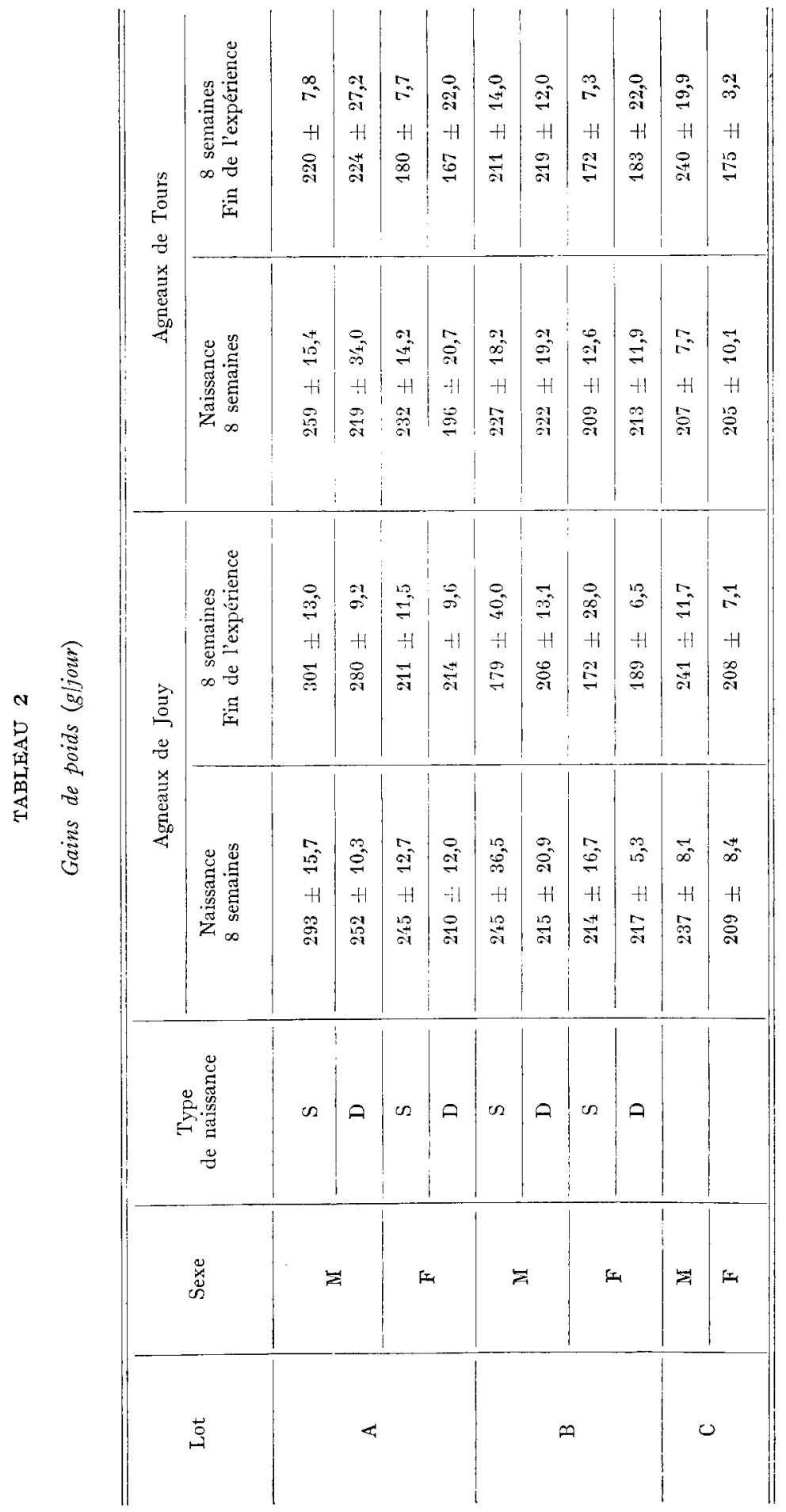


à 8 semaines inférieure à celle des agneaux du lot $\mathrm{A}$ (cette différence n'étant pas significative) ce qui indique un mauvais appariement des lots; il n'y a pas eu de différence à Tours.

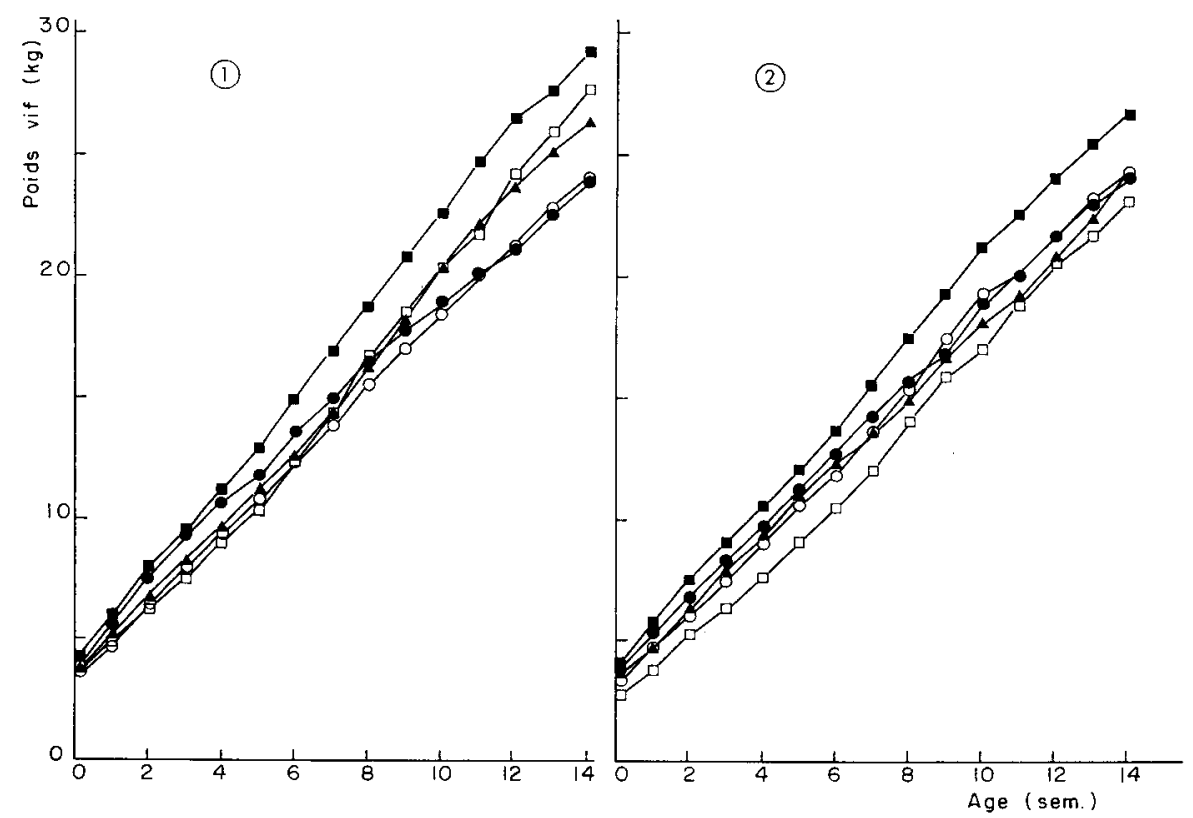

FIG. I. - Gains de poids cumulés des agneaux de Jouy (1) et de Tours (2)

Lot $A \begin{gathered}\text { Agneaux simples } \\ \text { Agneaux doubles }\end{gathered} \quad$ Lot $B \underset{\text { Agneaux doubles }}{\text { Agneaux }} \circ$ Lot $C$ A

Les agneaux allaités artificiellement et sevrés à 6 semaines (lot C) ont effectué des gains de poids de la naissance à 8 semaines légèrement inférieurs à Jouy $(225 \mathrm{~g} /$ jour $)$ comme à Tours ( $206 \mathrm{~g} / \mathrm{jour}$ ) à cetux des agneaux doubles des lots A ( 235 et $205 \mathrm{~g} /$ jour) et $\mathrm{B}(2 \mathrm{I} 6$ et $2 \mathrm{I} 7 \mathrm{~g} / \mathrm{jour})$.

Le sevrage a sensiblement ralenti la croissance des agneaux du lot $B$; les agneaux mâles ont effectué jusqu'à l'abattage des gains de poids inférieurs à ceux des agneaux correspondants du lot $\mathrm{A}$, notamment à Jouy où cette différence a été significative pour les simples $(P \leqslant 0,05)$ comme pour les doubles $(P \leqslant 0,0 r)$.

En revanche, les agneaux mâles dı lot $\mathrm{C}$ ont etı pendant la phase d'engraissement une vitesse de croissance supérieure à celle qu'ils avaient eue avant 8 semaines, en particulier à Tours où ils ont même effectué des gains de poids supérieurs à cetux des agneaux élevés sous la mère (lot A). A Jouy, comme à Tours, leur croissance a été plus rapide que celle des agneaux correspondants du lot $\mathrm{B}$.

Il faut enfin remarquer que, à Jouy comme à Tours, les différences entre agneaux d'un même lot ont été beaucoup moins marquées pour les agneaux élevés artificiellement (lot $\mathrm{C}$ ) que pour les agneaux élevés sous la mère (lot $\mathrm{A}$ ) et surtout que pour les agneaux sevrés précocement (lot $\mathrm{B}$ ). 


\section{Carcasses}

Les carcasses provenant des trois lots ont été jugées de bonne qualité. Les différences entre les lots de 'Tours ont été très faibles (tabl. 3) ; en revanche à Jouy la conformation des carcasses provenant des agneaux sevrés précocement (lot B) a été sensiblement moins bonne, tandis que les carcasses des agneaux élevés sous la mère (lot A) étaient plus lourdes et comportaient un poids de graisse périrénale significatirement $(\mathrm{P} \leqslant 0,00 \mathrm{I})$ plus élevé que celles des lots $\mathrm{B}$ et $\mathrm{C}$.

Les trois demi-carcasses de chaque lot qui ont été disséquées (tabl. 4) correspondaient à des agneaux ayant effectué des gains de poids voisins de la moyenne de leurs lots respectifs (cependant les poids des carcasses provenant des lots A et B ont été sensiblement inférieurs à la moyenne de ces lots). La proportion de tissu adipeux dans la demi-carcasse a été plus élevée pour le lot $\mathrm{A}(20,2 \mathrm{p}$. roo) que pour les lots $\mathrm{B}$ (I 7,I p. roo) et C (I6,2 p. IOo) ; les mêmes différences sont apparues dans la composition du gigot. Ces observations confirment les différences d'état d'engraissement appréciées sur l'ensemble des agneaux abattus par le poids de la graisse périrénale.

\section{Quantités d'aliments ingérées}

Les quantités de "lait "ingérées par les agneaux du lot $\mathrm{C}$ ont atteint à Jouy I $\mathrm{r} 20 \mathrm{ml}$ en moyenne par agneau et par jour dès la $2^{\mathrm{e}}$ semaine, et sont alors demeurées relativement constantes jusqu'à 4 semaines. En revanche à Tours, les quantités ingérées ont augmenté régulièrement jusqu'à la $4^{\mathrm{e}}$ semaine, oì elles ont atteint en moyenne I 2ro $\mathrm{ml}$ par agneau et par jour. De l'entrée en expérience au sevrage à 6 semaines, les agneaux de Jouy ont ingéré en moyenne $986 \mathrm{ml}$ de "lait " par tête et par jour, et ceux de Tours I $036 \mathrm{ml}$; cette différence n'a pas été significative.

Les quantités d'aliment concentré et de foin ingérées par les trois lots ont augmenté avec l'âge des animaux, cette évolution étant plus irrégulière à Tours, en raison de la qualité variable du foin (fig. 2). Les agneaux du lot B ont ingéré jusqu'à 8 semaines sensiblement moins d'aliment concentré que les agneaux du lot $\mathrm{A}$; le sevrage a entraîné un accroissement considérable de l'ingestion d'aliment concentré à Jouy, de foin à Tours.

La réduction, dès la $5^{\mathrm{e}}$ semaine, du nombre de ;distributions de "lait " aux agneaux du lot $\mathrm{C}$ a entrainé une augmentation rapide et régulière des quantités d'aliments solides ingérées, qui ont été dès lors bien supérieures à celles consommées par les autres lots ; cette différence a été particulièrement importante pour les quantités d'aliment concentré ingérées à Jouy.

Les quantités totales d'aliment concentré ingérées de o à 8 semaines ont été relativement voisines pour les trois lots à Jouy $(4,4 ; 3,9$ et $5,3 \mathrm{~kg}$ respectivement pour les lots $\mathrm{A}, \mathrm{B}$ et $\mathrm{C})$, et surtout à Tours $(6,0 ; 5,9$ et $5,2 \mathrm{~kg})$; ces différences ont été plus importantes pour le foin : 2,$2 ; \mathrm{r}, 7$ et $6,6 \mathrm{~kg}$ à Jouy et 2,$8 ; 3,7$ et $4,7 \mathrm{~kg}$ à Tours.

L,es quantités ingérées pendant la phase d'engraissement n'ont pas pu être mesurées jusqu'à la fin de l'expérience ; elles ne peuvent donc être comparées, car les différences entre les lots ont été dues essentiellement à la durée variable de cette phase d'engraissement, les agneaux des trois lots ayant été retirés de l'expérience au même poids. 


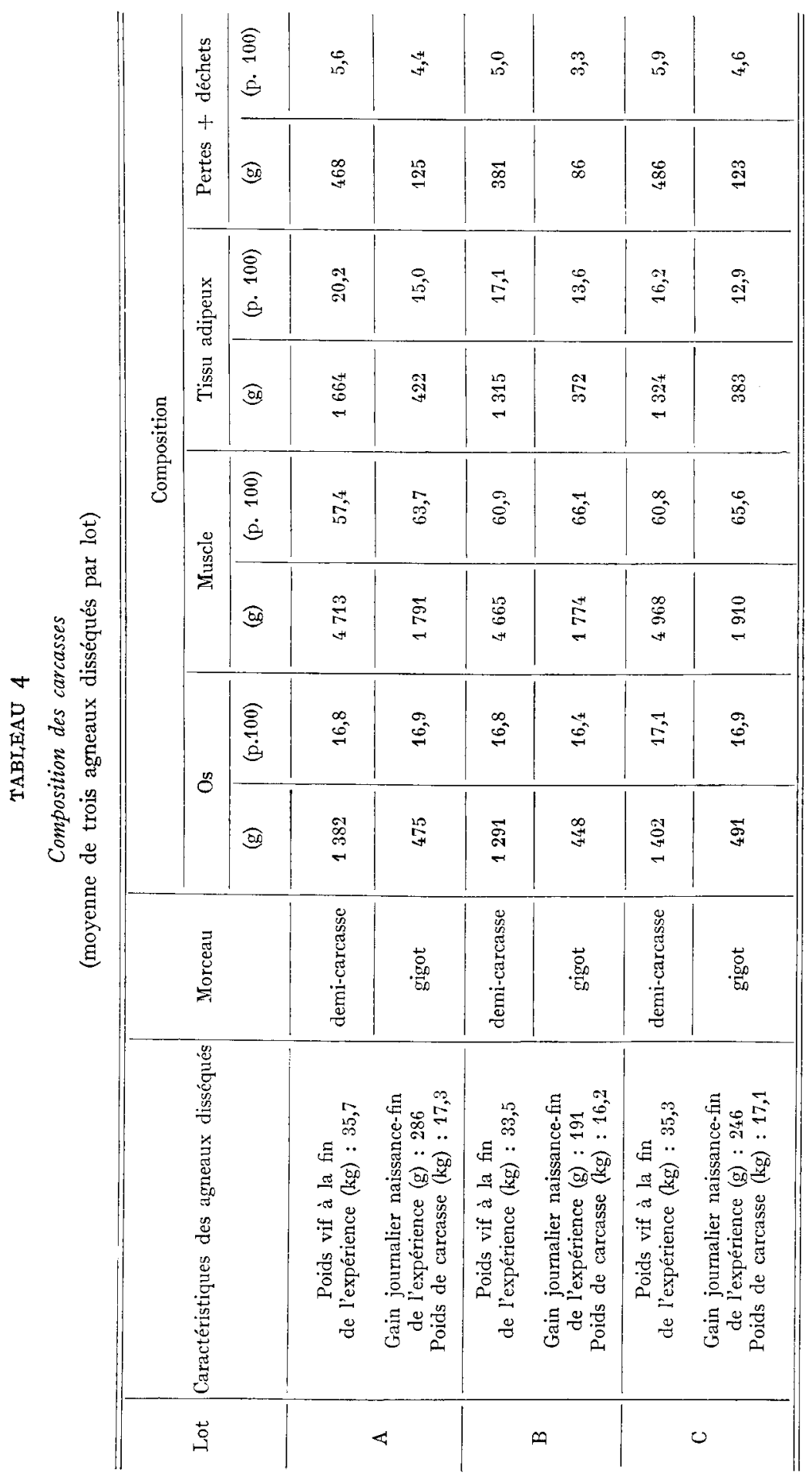


Sur l'ensemble de l'expérience (jusqu'à I4 semaines à Jouy, I 2 semaines à Tours), 1'aliment concentré a représenté $67 ; 62$ et 53 p. roo des aliments solides ingérés respectivement par les lots $\mathrm{A}, \mathrm{B}$ et $\mathrm{C}$, cette proportion ayant été pour les trois lots plus faible à Tours qu'à Jouy.
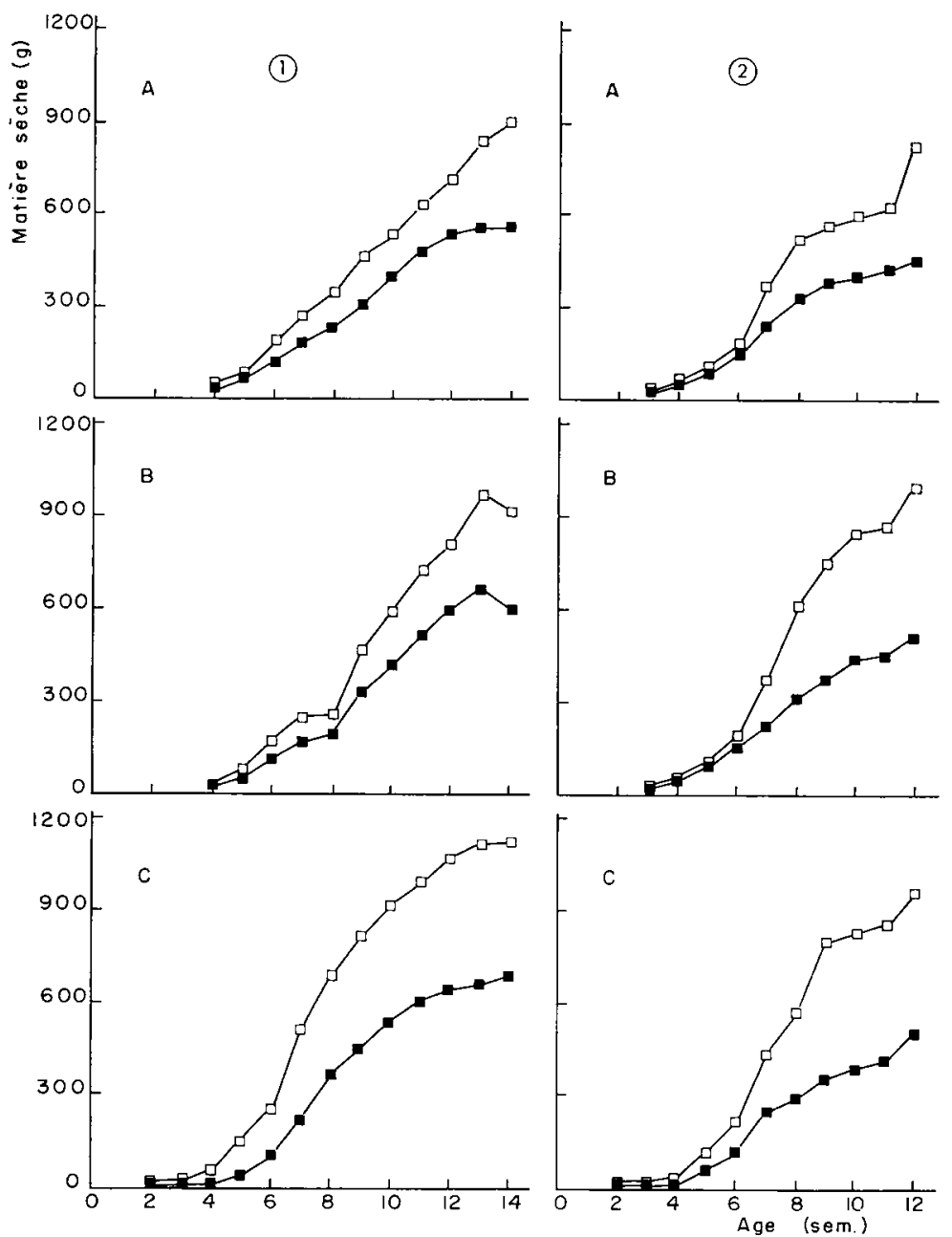

FIG. 2. - Evolution de la quantité de matière sèche ingérée, à Jouy $(I)$ et à Tours (2) sous forme d'aliment concentré (-) et de foin ( $\square$ ) (moyennes hebdomadaires)

Les quantités de matière sèche totale ingérées sous forme d'aliments solides, rapportées au poids vif pour chaque semaine de l'expérience, ont augmenté très régulièrement pour les agneaux $\mathrm{d} \mathfrak{u}$ lot $\mathrm{A}$ de Jouy (fig. 3) ; pour les agneaux du lot $\mathrm{B}$, elles ont augmenté plus rapidement après le sevrage, en se situant cependant jusqu'à $1 \mathrm{a} \mathrm{I} 3^{\mathrm{e}}$ semaine à un niveau très largement inférieur à celui atteint par les agneaux du lot C.

A Tours, les agneaux du lot $\mathrm{A}$ ont atteint dès la $8^{\mathrm{e}}$ semaine un niveau élevé 
d'ingestion de matière sèche $(3 \mathrm{~kg} /$ Ioo $\mathrm{kg}$ de poids vif). Les agneaux des lots $\mathrm{B}$ et $\mathrm{C}$ ont suivi une évolution très voisine, et atteignaient à 9 semaines le même niveau de consommation que les agneaux du lot $\mathrm{C}$ de Jouy.

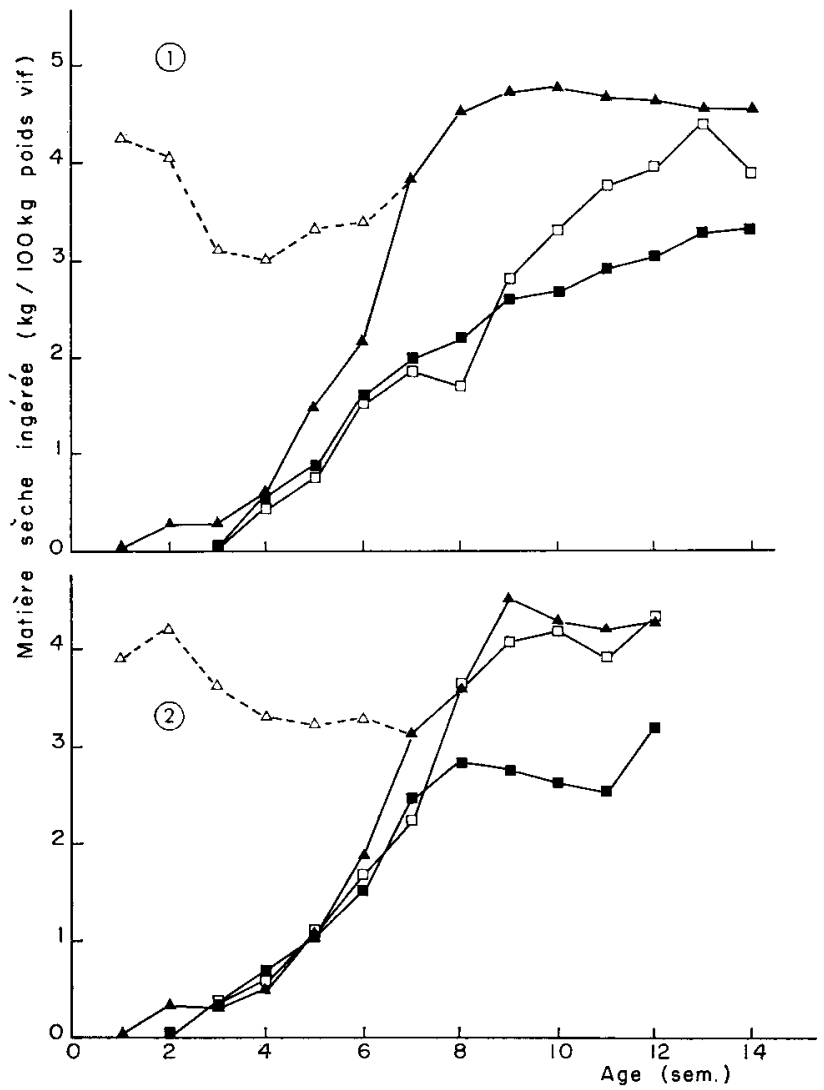

FIG. 3. - Évolution de la quantité de matière sèche ingérée sous forme d'aliments solides, exprimée par rapport au poids vit, d̀ Jouy (1) et à Tours (2) par les agneaux des lots $A(\mathbf{\bullet}), B(\square)$ et $C(\mathbf{\Lambda})$, et de la matière sèche totale ingérée par les agneaux du lot $C(\triangle)$ (moyennes hebdomadaires).

\section{DISCUSSION}

Le caractère annexe de cet essai ne nous a pas permis de comparer trois lots strictement homologues d'animaux. Les résultats obtenus permettent cependant d'une part de confirmer l'intérêt de la technique d'élevage artificiel des agneaux, d'autre part de comparer de manière globale les trois modes d'élevage utilisés.

\section{Intérêt de l'élevage artificiel des agneaux}

Les agneaux élevés artificiellement (lot $\mathrm{C}$ ) ont effectué des gains de poids très satisfaisants à Jouy comme à Tours ; les agneaux mâles ont atteint le poids de $35 \mathrm{~kg}$ à l'âge de $I 32$ jours à Jouy, de $I 38$ jours à Tours. Ils ont constitué des lots très homogènes, puisque l'allaitement artificiel a permis aux agneaux doubles d'ingérer autant 
de " lait " que les agneaux simples et d'effectuer ainsi une croissance comparable, et que les difficultés sanitaires ont été très réduites.

Les gains de poids des agneaux mâles pendant la période d'allaitement $(237 \mathrm{~g} / \mathrm{j}$ à Jouy ; $207 \mathrm{~g} / \mathrm{j}$ à Tours) sont très comparables à ceux que nous avions obtenus auparavant avec des agneaux de même race, recevant le même aliment d'allaitement dilué à raison de $\mathrm{I} 80 \mathrm{~g} /$ litre $(22 \mathrm{I} \mathrm{g} / \mathrm{j}$ de o à 9 semaines; PINOT et TÉISSIER, I965), ou de r75 et de $200 \mathrm{~g} /$ litre ( 2 I2 et $235 \mathrm{~g} / \mathrm{j}$ de o à 9 semaines ; PINot, I966). Ils sont cependant nettement inférieurs à ceux obtenus par LARGE (I965 $b$ ) avec des agneaux Dorset Horn $(280-300 \mathrm{~g} / \mathrm{j})$, qui avaient ingéré des quantités de "lait " beaucoup plus élevées.

Dans un essai antérieur (PINot et Téssier, I965), où le "lait " était distribué à volonté en quatre repas par jour jusqu'à six semaines, les quantités d'aliments solides ingérées avaient augmenté relativement peu de la $3^{\mathrm{e}}$ à la $6^{\mathrm{e}}$ semaine. En revanche dans le présent essai, la réduction progressive du nombre de distributions de " lait " et le sevrage à 6 semaines, ont entraîné un accroissement régulier des quantités d'aliments solides ingérées, et ont permis d'éviter toute réduction des gains de poids vif au moment du sevrage. Cependant, la vitesse de croissance n'a pas augmenté sensiblement pendant la phase d'engraissement, à l'inverse de ce qui avait été observé lors de ce premier essai (Pinó et TEIssie, I965).

\section{Comparaison entre les trois modes d'élevage des agneaux}

Les agneaux élevés sous la mère (lot $A$ ) ont effectué des gains de poids plus élevés que ceux des deux autres lots, et ont fourni une carcasse de meilleure qualité. Les agneaux élevés artificiellement ont eu eux aussi une croissance régulière, quoique moins rapide ; ils ont donné des carcasses de bonne qualité, au prix d'une ingestion d'aliments solides largement supérieure pendant la phase d'engraissement. Enfin les agneaux du lot $\mathrm{B}$ ont mal supporté le choc du sevrage, et se sont classés nettement derrière ceux des autres lots.

Après le sevrage, les agneaux du lot B n'ont pas pu atteindre le même niveau d'ingestion que les agneaux élevés artificiellement, tout au moins à Jouy. En effet les modalités du sevrage ont été sensiblement différentes pour les deux lots : d'une part, pour les agneaux du lot $B$, il a été lié à la séparation de la mère ; d'autre part, la réduction des quantités de "lait " distribuées aux agneaux du lot $\mathrm{C}$ a été régulière. Il semble ainsi que le maintien d'une vitesse de croissance élevée au moment du sevrage soit lié au passage progressif de l'alimentation liquide à l'alimentation solide. Afin de déterminer les modalités optimum du sevrage des agneaux sous la mère, il serait nécessaire d'étudier la relation entre les quantités de lait et d'aliments solides ingérées par ces agneaux comme il a été fait dans le cas de l'agneau élevé artificiellement (SPEdding et al., Ig62) et dans celui du veau (MATHIEU et WEGAT-IiTRE, I96r).

Iorsque les conditions d'élevage permettent aux agneaux sous la mère d'effectuer une croissance rapide (troupeau de Jouy), les agneaux élevés artificiellement réalisent des gains de poids plus faibles et ingèrent une quantité d'aliments supérieure. Lorsque les conditions sont moins favorables (troupeau de Tours), les différences entre ces deux modes d'élevage sont beaucoup moins accentuées. 


\author{
SUMMARY \\ ARTIFICIAI, REARING OF LAMBS \\ II. - COMPARISON BETWEEN THREE REARING METHODS
}

Three methods of lamb rearing were compared, in two flocks : lambs suckled and weaned at I 20 days; lambs suckled and weaned at eight weeks; lambs artificially reared from the 2 nd to 4 th day after birth, and weaned at six weeks.

The artificially reared lambs were given a replacement milk reconstituted from a powder including $70 \mathrm{p}$. I00 dried skim milk and $30 \mathrm{p}$. I 00 tallow, diluted to $200 \mathrm{~g} /$ litre. The lambs of the three groups were kept indoors, with lucerne hay and freely given concentrate. Most of the male lambs were slaughtered at $35 \mathrm{~kg}$.

The artificially reared lambs had before weaning growth rates as high as naturally suckled twins (table 2) ; they suffered no check after weaning, and yielded as good carcasses as the normally suckled lambs. However, the early weaned lambs had much lower growth rates during the fattening than during the suckling period, and yielded lower quality carcasses.

It is therefore possible to obtain good quality lambs by artificial rearing, and an early, but progressive weaning.

\title{
RÉFÉRENCES BIBLIOGRAPHIQUES
}

Boccard R., Dumont B.-L., 195. Étude de la production de la viande chez les ovins. I. Définition d'une découpe de référence. Ann. Zoolech., 4, 241-257.

Boccard R., Dumont B.-L., ig6o. Note sur la mesure et la variation de l'adiposité des carcasses d'agneaux. VI. Réunion des Instituls de Recherches sur les Viandes, Utrecht.

Brown T.-H., I964. The early weaning of lambs. J. Agric. Sci., 63, 191-204.

Charlet Lery G., Leroy A.-M., Zelter S.-Z., 1954. Élevage artificiel de l'agneau précocement sevré. Essai de détermination de ses dépenses de croissance. Ann. Zoolech., 3, 169-187.

Cunningiam J.-M.-M., Edwards R.-A., Simpson M.-E., i96r. Rearing lambs on a synthetic diet Animal, Prod̆., 3, 105-109.

LARGE R.-V., r965a. The artificial rearing of lambs. J. Agric. Sci., 65, ror-ro8.

LARGE R.-V., I $965 b$. The effect of concentration of milk substitute on the performance of artificially reared lambs. Animal, Prod. $7,3^{25}-33^{2}$.

Mathieu C.-M., Wegat-Litre Ema, ig6r. Mise au point d'une méthode d'alimentation des veaux d'élevage. I. Détermination de la quantité de lait nécessaire. Ann. Zootech., 10, I6I-1 75 .

Mauleon P., Pinot R., Du Mesnil du Buisson F., i965. Perspectives nouvelles de réussite de deux agnelages par an chez la brebis. Bull. C. E. T. A., n ${ }^{\circ}$ I062.

Pinot R., Teissier J.-II., i 965. L'allaitement artificiel des agneaux. I. Comparaison entre différents laits de remplacement et le lait de brebis. Ann. Zoolech., 14, 26r-278.

Pinot R., I 966 . Résultats non publiés.

Pribicevic S., Jovanovic M., Sokolic A., Cuperlovic K., Movsesijan R., Stojanovic N., ig6z. Artificial rearing of lambs. Vel. Glasn., 16, 737-742.

SPEDDing C.-R.-W., Brown T.-H., LARGE R.-V., I 1963 . The effect of milk intake on nematode infestation of the lamb. Proc. Nutr. Soc., 22, 32-41.

Welcit J.-G., Van Der Noot G.-W., Gilbreath R.-L., I963. Effect of feeding milk replacers with varying amounts of fat for hothouse lamb production. J. Anim. Sci., 22, I $55^{-1} 5^{8}$. 